\title{
Plant growth regulators involved in abiotic stress: preface
}

\author{
Stefaan P. O. Werbrouck ${ }^{1} \cdot$ Guoping Zhang $^{2}$
}

Published online: 31 March 2015

(C) Springer Science+Business Media Dordrecht 2015

An ever-expanding world population and globally changing diet preferences have put considerable pressure on the worldwide agricultural community to produce more food, feed and bio-energy crops. As a result, marginal land areas will need to be used to meet the increasing requirement of future generations, especially in developing countries. These marginal areas commonly impose abiotic stresses on crops due to factors such as salinity, drought, temperature extremes, flooding, low nutrients and aluminium or heavy metal toxicity. As a consequence, the growth and yield of crops from such areas is typically low and their quality poor, limiting farmer income. Therefore, it is important to gain knowledge and insight of the intrinsic flexibility by which plants respond to these abiotic stresses. Throughout evolution, plants have developed a dazzling array of complex strategies for coping with abiotic stresses and adapting to a wide range of environments. Biochemical, physiological and molecular genetic studies of these strategies have been initiated, and, as in many plant research fields, those on Arabidopsis pave the way. However, the contributions in this special issue illustrate the progress made on other species and highlight the insights into this research field.

The calcineurin B-like (CBL) and CBL-interacting protein kinase (CIPK) signalling pathway is a flexible, $\mathrm{Ca}^{2+}$ signalling network that allows plants to fine-tune their stress mitigation responses. Thoday-Kennedy et al. (2015) provide a critical account of our current knowledge of CBL and CIPK interactions and their roles in ion

Stefaan P. O. Werbrouck

Stefaan.Werbrouck@UGent.be

1 Ghent University, Ghent, Belgium

2 Zhejiang University, Hangzhou, People's Republic of China transport under abiotic stress. Han et al. (2014) review the signalling pathways and key mechanisms of sodium detoxification mediated by ion transporters and antiporters, and give an overview of practical strategies and methodologies for functional characterisation of high-affinity potassium transporters. In addition, they discuss how to alleviate salt stress through the exogenous application of gibberellins and nitric oxide. Li et al. (2014a) focus on salt cress (Thellungiella halophila), a close relative of Arabidopsis, a species that has emerged as a model halophyte for the molecular elucidation of abiotic stress tolerance. Its seed germination is highly regulated by environmental and hormonal signals; thus, it provides opportunities to study how such external conditions are sensed. The mung bean (Vigna radiata) is salinity-sensitive and suffers considerably from soil salinity; as a consequence, reductions in germination, chlorophyll content, vigour and yield have been reported. Patel et al. (2014) draw attention to interactions that occur with this species in the rhizosphere and report on those with halotolerant Bacillus spp. These species were isolated from saline habitats and alleviate salt stress in mung beans thereby increasing germination and partially restoring root and shoot growth. Xuan et al. (2014) characterise the response of seedlings of yellowand black-seeded rape to salt and cadmium stresses, and show how tolerance is associated with flavonoid content. $\mathrm{Li}$ et al. (2014b) demonstrate how improvement of endogenous polyamine levels by exogenous spermidine application may promote drought tolerance of plants.

Waterlogging is also a major constraint to crop production in many parts of the world due to anoxic conditions in the root zone. Najeeb et al. (2015) investigate the mechanisms of waterlogging tolerance in cotton induced by aminoethoxyvinylglycine and show the how leaf growth and fruit production can be enhanced under these 
conditions. Jayakannan et al. (2015) review the role of salicylic acid (SA) in the regulation of ion transport processes during salt stress. They summarise current knowledge of SA biosynthesis and metabolism, and discuss its transport, sensing and interactions with other plant hormones and signalling molecules. High concentrations of heavy metals, such as cadmium, in plants cause oxidative stress due to the accumulation of reactive oxygen species. In this respect, Singh and Shah (2015) show that SA signalling plays an integrating role in rice seedlings in mitigating Cd-induced toxicity.

We hope that this special issue focusing on abiotic stresses will further stimulate research on these challenging problems. An increased understanding of stress sensing, signalling networks and interactions among plant hormones will provide more possibilities for improving crop yield through applications of plant growth regulators or through genetic modifications. Finally, we would like to thank contributors and reviewers for offering us the benefit of their knowledge, and to Plant Growth Regulation for giving us the opportunity to present this special issue.

\section{References}

Han Y, Yin S, Huang L (2014) Towards plant salinity toleranceimplications from ion transporters and biochemical regulation. Plant Growth Regul. doi:10.1007/s10725-014-9997-6
Jayakannan M, Bose J, Babourina O, Rengel Z, Shabala S (2015) Salicylic acid in plant salinity stress signalling and tolerance. Plant Growth Regul. doi:10.1007/s10725-015-0028-z

Li W, Khan MA, Yamaguchi S, Liu X (2014a) Hormonal and environmental regulation of seed germination in salt cress (Thellungiella halophila). Plant Growth Regul. doi:10.1007/ s10725-014-0007-9

Li Z, Zhou H, Peng Y, Zhang X, Ma X, Huang L, Yan Y (2014b) Exogenously applied spermidine improves drought tolerance in creeping bentgrass associated with changes in antioxidant defense, endogenous polyamines and phytohormones. Plant Growth Regul. doi:10.1007/s10725-014-9978-9

Najeeb U, Atwell BJ, Bange MP, Tan DK (2015) Aminoethoxyvinylglycine (AVG) ameliorates waterlogging-induced damage in cotton by inhibiting ethylene synthesis and sustaining photosynthetic capacity. Plant Growth Regul. doi:10.1007/s10725-0150037-y

Patel RR, Patel DD, Thakor P, Patel B, Thakkar VR (2014) Alleviation of salt stress in germination of Vigna radiata L. by two halotolerant Bacilli sp. isolated from saline habitats of Gujarat. Plant Growth Regul. doi:10.1007/s10725-014-0008-8

Singh I, Shah K (2015) Evidences for suppression of cadmium induced oxidative stress in presence of sulphosalicylic acid in rice seedlings. Plant Growth Regul. doi:10.1007/s10725-0150023-4

Thoday-Kennedy EL, Jacobs AK, Roy SJ (2015) The role of the CBL-CIPK calcium signalling network in regulating ion transport in response to abiotic stress. Plant Growth Regul. doi:10. 1007/s10725-015-0034-1

Xuan L, Hussain N, Wang Z, Jiang Y, Chen M, Jiang L (2014) Comparison of vitality between seedlings germinated from black-coated and yellow-coated seeds of a turnip rape (Brassica rapa L.) subjected to $\mathrm{NaCl}$ and $\mathrm{CdCl}_{2}$ stresses. Plant Growth Regul. doi:10.1007/s10725-014-0019-5 